\title{
TRANSVERSE SHEAR EFFECT IN A CIRCUMFERENTIALLY CRACKED CYLINDRICAL SHELL*
}

\author{
BY \\ F. DELALE AND F. ERDOGAN \\ Lehigh University
}

\begin{abstract}
The problem of a cylindrical shell containing a circumferential through crack is considered by taking into account the effect of transverse shear deformations. The formulation is given for a specially orthotropic material within the confines of a linearized shallow shell theory. The particular theory used permits the consideration of all five boundary conditions regarding moment and stress resultants on the crack surface. The stress intensity factors are calculated separately for a cylinder under a uniform membrane load, and that under a uniform bending moment for values of $0 \leq \lambda_{2}=\left[12\left(1-v^{2}\right)\right]^{1 / 4}$. $a(R h)^{1 / 2} \leq 10$ and $0<a / h \leq 10$, where $2 a, h$, and $R$ are, respectively, the crack length, the thickness, and the (mean) radius of the cylinder. Sample results showing the nature of the out-of-plane crack surface displacement and the effect of the Poisson's ratio are also presented.
\end{abstract}

1. Introduction. Depending on the mechanical behavior of the material and the nature of the loading and environmental conditions, in designing line pipes and cylindrical containers it is often necessary to consider fatigue crack propagation and fracture among the possible modes of failure. This requires, in addition to the application of standard techniques in the failure analysis of the component, the treatment of the problem from the viewpoint of fracture mechanics. In using this approach the initial flaws or certain types of imperfections which may exist in the material are treated as "cracks". These cracks may propagate under fluctuating external loads as fatigue cracks, or under sustained loads and sufficiently adverse environmental conditions as stress corrosion cracks or as a combination of both, namely as fatigue corrosion cracks.

Up to now, fracture mechanics research as applied to pipeline and container safety has dealt almost exclusively with longitudinal flaws in the component. There is, of course, a good reason for this, namely that in pressurized cylinders under normal operating conditions, the hoop stress is by far the dominant stress component. On the other hand, any secondary loads caused by known and unforeseen factors would be primarily in the axial direction. These are mostly bending and axial constraint stresses. Some of the sources of these stresses are support misalignment, variety of thermal fluctuations, ground settlement and earthquakes, hydroelastic or aeroelastic loading, axial constraint in buried pipes, gross bending in offshore piping caused by a variety of oceanic disturbances or generated

* Received August 3, 1978; revised version received November 20, 1978. This study was partially supported by the National Science Foundation under Grant ENG78-09737, by NASA-Langley under Grant NGR-39007-011, and by the Department of Transportation under Contract DOT-RC-82007. 
during installation, stresses arising from vehicular vibrations and impact, and the residual stresses in and around girth welds. One may also point out that the axial stresses would be much more time-varying than basically pressure-induced hoop stresses and hence would tend to facilitate the formation and propagation of fatigue cracks in the circumferential direction. If the thickness-to-radius ratio in the cylinder is sufficiently small, then a bending theory of shells may be used to formulate the circumferential crack problem and, by approximating the internal stresses (such as residual and thermal stresses) by a linear function in the thickness coordinate, the general solution may be obtained by appropriately superimposing the results of membrane and bending solutions. The existing solutions of this problem take into account the membrane loading only and are based on an eighth-order shallow-shell theory in which the effect of the transverse shear stress is ignored and the crack surface boundary conditions are approximated by using the Kirchhoff assumption' [1-3]. The consequence of this assumption in shells is the same as in the bending of cracked flat plates [4-7]. It has been shown that if a plate bending theory compatible with the number of independent boundary conditions is not used to solve the crack problem, the computed stress intensity factors may contain errors and the angular distribution of membrane and bending stress resultants would be different [4-7]. This would then make it difficult to justify the superposition of membrane and bending solutions in fatigue crack propagation and fracture analysis.

The aim of this paper is to solve the problem of a circumferentially-cracked cylindrical shell by taking into account the effect of transverse shear and to obtain the stress intensity factors for the bending moment as well as the membrane force as the external load. The problem for a longitudinal crack was recently solved [8] by using a higher-order shallow shell theory described in [9]. In the present paper the effect of Poisson's ratio on the stress intensity factors and the nature of the out-of-plane displacement along the edges of the crack (i.e., bulging) will also be studied.

2. Formulation of the problem. In this problem the general shallow shell equations developed in [9] for an isotropic medium will be used. However, as shown in [10] and [11] for the classical shell theory, in this case too it can be shown that the related differential equations of the orthotropic shells can be factorized and reduced to the equations of isotropic shells if one assumes the material to be "specially orthotropic".

Following [8] and [9], the equilibrium equations for a shallow shell may be expressed as

$$
\begin{gathered}
N_{i, j}=0, \quad V_{i, i}+\left(Z_{i} N_{i j}\right)_{j}+q\left(X_{1}, X_{2}\right)=0, \\
M_{i, j}-V_{i}=0 \quad(i=1,2, j=1,2)
\end{gathered}
$$

where $N_{i j}, M_{i j}$, and $V_{i}(i, j=1,2)$ are, respectively, membrane, moment and transverse shear resultants, and the indicial notation and the summation convention are used. The components of strains are given by

$$
\varepsilon_{i j}=\frac{1}{2}\left[U_{i, j}+U_{j, i}+Z_{. i} W_{j}+Z_{j} W_{.,}\right] \quad(i, j=1,2)
$$

\footnotetext{
' That is, on the traction-free crack surface instead of satisfying the conditions $M_{n s}=0, V=0$ separately, the condition $V+\partial M_{n s} / \partial s=0$ is satisfied, thereby making the number of boundary conditions (four) and the order of the reduced differential equation (eighth) compatible. Here $n$ and $s$ are the coordinates normal and parallel to the crack, $M_{n s}$ is the twisting moment, and $V$ is the transverse shear.
} 
where $U_{1}, U_{2}$ and $W$ are, respectively, $X_{1}, X_{2}$, and $Z$-components of the displacement vector, and the function $Z\left(X_{1}, X_{2}\right)$ giving the equation of the middle surface is known. Let $\beta_{1}$ and $\beta_{2}$ be the angles of rotation of the normal to shell surface. The (transverse) shear strains may then be expressed as

$$
\theta_{i}=W_{, i}+\beta_{i}, \quad i=1,2 .
$$

With Hook's law

$$
\varepsilon_{i j}=a_{i j k l} N_{k l} / h
$$

and the relations giving $M_{i j}$ and $V_{i}$ in terms of $\beta_{i}$ and $\theta_{i}$, respectively, the formulation of the problem would be technically complete, the ten equations (2.1)-(2.5) accounting for the ten variables $\varepsilon_{i j}, \beta_{i}, \theta_{i}, U_{i}$, and $W$.

First, eliminating $U_{1}$ and $U_{2}$ from (2.4), one obtains the following compatibility equation:

$$
e_{i k} e_{j r}\left(\varepsilon_{i j, k l}+Z_{, i j} W_{, k l}\right)=0 .
$$

where $e_{i k}$ is the permutation symbol ${ }^{2}$. Next, defining the stress function $F\left(X_{1}, X_{2}\right)$ by

$$
N_{i j}=e_{i k} e_{j l} F_{, k l}
$$

it is seen that (2.1) is satisfied and (2.2) and (2.7) reduce to

$$
\begin{gathered}
M_{i j, i j}+Z_{. i j} e_{i k} e_{j l} F_{. k l}+q=0, \\
e_{i m} e_{j n} e_{k p} e_{l q} a_{i j k l} F_{, m n p q}+h Z_{, i j} e_{i k} e_{j l} W_{, k l}=0 .
\end{gathered}
$$

Even with simple shell geometries for anisotropic materials the differential equations are not tractable. However, as in [10] if one assumes a special orthotropy, the related differential operators in these equations can be factorized and the problem can be made analytically tractable. Let the material be orthotropic and the stress strain relations be

$$
\begin{array}{ll}
\varepsilon_{11}=\frac{1}{h E_{1}}\left(N_{11}-\nu_{1} N_{22}\right), & \varepsilon_{12}=N_{12} / 2 h G_{12}, \\
\varepsilon_{22}=\frac{1}{h E_{2}}\left(N_{22}-\nu_{2} N_{11}\right), & \nu_{1} / E_{1}=\nu_{2} / E_{2}
\end{array}
$$

In this case too the following is the condition for the factorization of the operators:

$$
2 G_{12}=\left(E_{1} E_{2}\right)^{1 / 2} /\left(1+\left(\nu_{1} \nu_{2}\right)^{1 / 2}\right)
$$

A material satisfying the condition (2.12) is said to be "specially orthotropic". It has been shown that for certain orthotropic materials the value of the shear modulus calculated from (2.12) and that measured experimentally are very nearly the same and consequently with the assumption of special orthotropy, the analysis can be simplified quite considerably [11]. If we now define

$$
E=\left(E_{1} E_{2}\right)^{1 / 2}, \quad \nu=\left(\nu_{1} \nu_{2}\right)^{1 / 2}, \quad c=\left(E_{1} / E_{2}\right)^{1 / 4}
$$

$$
{ }^{2} e_{11}=0=e_{22}, e_{12}=1=-e_{21} \text {. }
$$


the stress-strain relations (2.11) become

$$
\varepsilon_{11}=\frac{1}{h E}\left(\frac{N_{11}}{c^{2}}-\nu N_{22}\right), \quad \varepsilon_{12}=\frac{1+\nu}{h E} N_{12}, \quad \varepsilon_{22}=\frac{1}{h E}\left(c^{2} N_{22}-\nu N_{11}\right) .
$$

From the linear thickness variation of the stress components $\sigma_{i j}$ one obtains

$$
\begin{gathered}
M_{11}=D\left(c^{2} \beta_{1,1}+\nu \beta_{2,2}\right), \quad M_{12}=\frac{D(1-\nu)}{2}\left(\beta_{1,2}+\beta_{2,1}\right), \\
M_{22}=D\left(\nu \beta_{1,1}+\beta_{2,2} / c^{2}\right), \quad D=\frac{E h^{3}}{12\left(1-\nu^{2}\right)} .
\end{gathered}
$$

Also, assuming a linear transverse shear stress-strain relationship, one finds

$$
\theta_{1}=(1 / \operatorname{ch} B) V_{1}, \quad \theta_{2}=(c / h B) V_{2},
$$

where $B$ is the effective shear modulus. Referring to [12], it will be assumed that

$$
B=\frac{5}{6} \frac{E}{2(1+\nu)} \text {. }
$$

Defining now the operator

$$
\nabla_{c}^{2}=c \frac{\partial^{2}}{\partial X_{1}^{2}}+\frac{\partial^{2}}{c \partial X_{2}^{2}},
$$

Eqs. (2.9), (2.10), and (2.3) may be reduced to

$$
\begin{gathered}
\nabla_{c}^{2} \nabla_{c}^{2} F+h E\left(\frac{\partial^{2} Z}{\partial X_{1}^{2}} \frac{\partial^{2}}{\partial X_{2}^{2}}-2 \frac{\partial^{2} Z}{\partial X_{1} \partial X_{2}} \frac{\partial^{2}}{\partial X_{1} \partial X_{2}}+\frac{\partial^{2} Z}{\partial X_{2}^{2}} \frac{\partial^{2}}{\partial X_{1}^{2}}\right) W=0, \\
D \nabla_{c}^{2} \nabla_{c}^{2} W-\left(1-\frac{D}{B h} \nabla_{c}^{2}\right)\left(\frac{\partial^{2} Z}{\partial X_{1}^{2}} \frac{\partial^{2}}{\partial X_{2}^{2}}-2 \frac{\partial^{2} Z}{\partial X_{1} \partial X_{2}} \frac{\partial^{2}}{\partial X_{1} \partial X_{2}}\right. \\
\left.+\frac{\partial^{2} Z}{\partial X_{2}^{2}} \frac{\partial^{2}}{\partial X_{1}^{2}}\right) F=\left(1-\frac{D}{B h} \nabla_{c}^{2}\right) q, \\
\beta_{1}+\frac{\partial W}{\partial X_{1}}=\frac{D}{h B}\left[\nabla_{c}^{2} \beta_{1}+\frac{1+\nu}{2 c} \frac{\partial}{\partial X_{2}}\left(\frac{\partial \beta_{2}}{\partial X_{1}}-\frac{\partial \beta_{1}}{\partial X_{2}}\right)\right], \\
\beta_{2}+\frac{\partial W}{\partial X_{2}}=\frac{D}{h B}\left[\nabla_{c}^{2} \beta_{2}+c \frac{1+\nu}{2} \frac{\partial}{\partial X_{1}}\left(\frac{\partial \beta_{1}}{\partial X_{2}}-\frac{\partial \beta_{2}}{\partial X_{1}}\right)\right] .
\end{gathered}
$$

Eqs. (2.19)-(2.22) provide the formulation for an arbitrary shallow shell in terms of the unknown functions $F, W, \beta_{1}$, and $\beta_{2}$.

Now let us assume that in the domain of interest the curvatures of the shell are constant. Then in (2.19) and (2.20) the terms involving $Z$ may be replaced by

$$
\frac{\partial^{2} Z}{\partial X_{1}^{2}}=-\frac{1}{R_{1}}, \quad \frac{\partial^{2} Z}{\partial X_{2}^{2}}=-\frac{1}{R_{2}}, \quad \frac{\partial^{2} Z}{\partial X_{1} \partial X_{2}}=-\frac{1}{R_{12}} .
$$

Also, following [8], if one introduces the dimensionless quantities given in Appendix A, Eqs. (2.19)-(2.22) may further be simplified as

$$
\nabla^{4} \phi-\frac{1}{\lambda^{2}}\left(\lambda_{1}{ }^{2} \frac{\partial^{2}}{\partial y^{2}}-2 \lambda_{12}{ }^{2} \frac{\partial^{2}}{\partial x \partial y}+\lambda_{2}{ }^{2} \frac{\partial^{2}}{\partial x^{2}}\right) w=0
$$




$$
\begin{gathered}
\nabla^{4} w+\lambda^{2}\left(1-\kappa \nabla^{2}\right)\left(\lambda_{1}^{2} \frac{\partial^{2}}{\partial y^{2}}-2 \lambda_{12}^{2} \frac{\partial^{2}}{\partial x \partial y}+\lambda_{2}^{2} \frac{\partial^{2}}{\partial x^{2}}\right) \phi=\lambda^{4}\left(1-\kappa \nabla^{2}\right) \frac{a}{h} q \\
\left(1-\kappa \nabla^{2}\right) \beta_{x}+\frac{\partial w}{\partial x}=\kappa \frac{1+\nu}{2} \frac{\partial}{\partial y}\left(\frac{\partial \beta_{y}}{\partial x}-\frac{\partial \beta_{x}}{\partial y}\right) \\
\left(1-\kappa \nabla^{2}\right) \beta_{y}+\frac{\partial w}{\partial y}=\kappa \frac{1+\nu}{2} \frac{\partial}{\partial x}\left(\frac{\partial \beta_{x}}{\partial y}-\frac{\partial \beta_{y}}{\partial x}\right)
\end{gathered}
$$

The constant $a$ used in Appendix A to normalize various quantities is a characteristic length parameter in the shell. Usually in crack problems the shell is assumed to be "infinitely large" and $a$ is taken to be half the crack length.

Defining now a new function $\Omega$ by

$$
\Omega(x, y)=\frac{\partial \beta_{x}}{\partial y}-\frac{\partial \beta_{y}}{\partial x}
$$

Eqs. (2.26) and (2.27) may be expressed as

$$
\beta_{x}=\kappa \nabla^{2} \beta_{x}-\frac{\partial w}{\partial x}-\kappa \frac{1+\nu}{2} \frac{\partial \Omega}{\partial y}, \quad \beta_{y}=\kappa \nabla^{2} \beta_{y}-\frac{\partial w}{\partial y}+\kappa \frac{1+\nu}{2} \frac{\partial \Omega}{\partial x},
$$

and it may easily be shown that

$$
\nabla^{2} \beta_{x}=\frac{\partial \Omega}{\partial y}+\frac{\partial^{2} \beta_{x}}{\partial x^{2}}+\frac{\partial^{2} \beta_{y}}{\partial x \partial y}, \quad \nabla^{2} \beta_{y}=-\frac{\partial \Omega}{\partial x}+\frac{\partial^{2} \beta_{x}}{\partial x \partial y}+\frac{\partial^{2} \beta_{y}}{\partial y^{2}} .
$$

Also, if we define the function $\psi$ by

$$
\psi(x, y)=\kappa\left(\frac{\partial \beta_{x}}{\partial x}+\frac{\partial \beta_{y}}{\partial y}\right)-w
$$

from (2.29) and (2.30) we obtain

$$
\beta_{x}=\frac{\partial \psi}{\partial x}+\kappa \frac{1-\nu}{2} \frac{\partial \Omega}{\partial y}, \quad \beta_{y}=\frac{\partial \psi}{\partial y}-\kappa \frac{1-\nu}{2} \frac{\partial \Omega}{\partial x} .
$$

In (2.32), eliminating $\Omega$ and then using (2.31), we find

$$
\kappa \nabla^{2} \psi-\psi-w=0 \text {. }
$$

Similarly, by eliminating $w,(2.29)$ yields

$$
\kappa \frac{1-\nu}{2} \nabla^{2} \Omega-\Omega=0
$$

The solution of the shell problem must then satisfy the differential equations (2.24), (2.25), (2.33) and (2.34) and all the necessary boundary conditions.

3. Cylindrical shell with a circumferential crack. Consider now a cylindrical shell containing a circumferential through crack of length $2 a$ as shown in Fig. 1. In this case $\lambda_{1}=0=\lambda_{12}$, and if we further assume that $q=0,(2.24)$ and (2.25) become

$$
\nabla^{4} \phi-\left(\lambda_{2} / \lambda\right)^{2} \frac{\partial^{2} w}{\partial x^{2}}=0
$$




$$
\nabla^{4} w+\left(\lambda \lambda_{2}\right)^{2}\left(1-\kappa \nabla^{2}\right) \frac{\partial^{2} \phi}{\partial x^{2}}=0
$$

Eliminating $\phi$, it follows from (3.1) and (3.2) that

$$
\nabla^{4} \nabla^{4} w+\lambda_{2}^{4}\left(1-\kappa \nabla^{2}\right) \frac{\partial^{4} w}{\partial x^{4}}=0
$$

The problem will be solved by using Fourier transforms. It will be assumed that through a proper superposition the original shell problem has been reduced to a perturbation problem in which self-equilibrating force and moment resultants acting on the crack surfaces are the only nonzero external loads (hence the assumption $q=0$ ). Thus, in some neighborhood of the crack in which the stresses are expected to be nonzero, the transform of $w$ and its inversion may be expressed as

$$
\begin{gathered}
f(x, \alpha)=\int_{-\infty}^{\infty} w(x, y) \exp (i \alpha y) d y, \\
w(x, y)=\frac{1}{2 \pi} \int_{-\infty}^{\infty} f(x, \alpha) \exp (-i \alpha y) d \alpha .
\end{gathered}
$$

Substituting from (3.5) into (3.3) one obtains an eighth-order ordinary linear differential equation in $f$. Looking for a solution in the form $f=R(\alpha) \exp (m x)$, the characteristic equation of the problem is found to be

$$
m^{8}-\left(\kappa \lambda_{2}^{4}+4 \alpha^{2}\right) m^{6}+\left(6 \alpha^{4}+\lambda_{2}^{4} \kappa \lambda_{2}^{4} \alpha^{2}\right) m^{4}-4 \alpha^{6} m^{2}+\alpha^{8}=0 .
$$

If we define

$$
p=m^{2}-\alpha^{2},
$$

Eq. (3.6) can be written in terms of $p$ as follows:

$$
p^{4}-\kappa \lambda_{2}^{4} p^{3}+\lambda_{2}^{4}\left(1-2 \kappa \alpha^{2}\right) p^{2}+\lambda_{2}^{4}\left(2 \alpha^{2}-\kappa \alpha^{4}\right) p+\lambda_{2}^{4} \alpha^{4}=0 .
$$

It should be emphasized that the roots of (3.8) are in general complex, and, of course, are not known as a function of $\alpha$ in closed form. After solving (3.8) let the roots of (3.6) be ordered such that

$$
\operatorname{Re}\left(m_{j}\right)<0, \quad m_{j+4}=-m_{j}, \quad j=1, \cdots, 4 .
$$

The solution $f(x, \alpha)$ of the resulting, ordinary differential equation satisfying the regularity conditions at $x=\mp \infty$ may then be expressed as

$$
\begin{aligned}
f(x, \alpha) & =\sum_{j=1}^{4} R_{j}(\alpha) \exp \left(m_{j} x\right), \quad x>0, \\
& =\sum_{j=5}^{8} R_{j}(\alpha) \exp \left(m_{j} x\right), \quad x<0 .
\end{aligned}
$$

If we let

$$
\phi(x, y)=\frac{1}{2 \pi} \int_{-\infty}^{\infty} g(x, \alpha) \exp (-i y \alpha) d \alpha,
$$

from (3.1), (3.2) and (3.10) we find 


$$
\begin{aligned}
g(x, \alpha) & =\left(\lambda_{2} / \lambda\right)^{2} \sum_{1}^{4}\left(m_{j} / p_{j}\right)^{2} R_{j}(\alpha) \exp \left(m_{j} x\right), & & x>0 \\
& =\left(\lambda_{2} / \lambda\right)^{2} \sum_{5}^{8}\left(m_{j} / p_{j}\right)^{2} R_{j}(\alpha) \exp \left(m_{j} x\right), & & x<0 .
\end{aligned}
$$

Similarly, assuming

$$
\Omega(x, y)=\frac{1}{2 \pi} \int_{-\infty}^{\infty} h(x, \alpha) \exp (-i y \alpha) d \alpha
$$

from (2.34) we obtain

$$
\begin{aligned}
h(x, \alpha) & =A_{1}(\alpha) \exp \left(r_{1} x\right), & & x>0 \\
& =A_{2}(\alpha) \exp \left(r_{2} x\right), & & x<0
\end{aligned}
$$

where

$$
r_{1}=-\left[\alpha^{2}+\frac{2}{\kappa(1-\nu)}\right]^{1 / 2}, \quad r_{2}=\left[\alpha^{2}+\frac{2}{\kappa(1-\nu)}\right]^{1 / 2} .
$$

Also, let us assume that

$$
\psi(x, y)=\frac{1}{2 \pi} \int_{-\infty}^{\infty} \theta(x, \alpha) \exp (-i y \alpha) d \alpha .
$$

it can be shown that the remaining differential equation (2.33) is satisfied if $\theta$ is assumed to be

$$
\begin{aligned}
\theta(x, \alpha) & =\sum_{1}^{4} \frac{R_{j}(\alpha)}{\kappa p_{j}-1} \exp \left(m_{j} x\right), & & x>0, \\
& =\sum_{5}^{8} \frac{R_{j}(\alpha)}{\kappa p_{j}-1} \exp \left(m_{j} x\right), & & x<0 .
\end{aligned}
$$

The expressions given by (3.10), (3.12), (3.14), and (3.17) satisfy the differential equations of the problem ${ }^{3}$. If one now determines the arbitrary functions $R j(\alpha)(j=1, \ldots, 8)$ and $A_{i}(\alpha)(i=1,2)$ in such a way that the boundary conditions of the problem are also satisfied, one then has the solution.

4. Boundary conditions. As mentioned earlier, the only external loads in the problem are the self-equilibrating force and moment resultants on the crack surfaces. These forces can be decomposed in such a way that in solving the problem one needs to consider (with respect to the $x=0$ plane) either symmetric or antisymmetric loading only. In these two cases the following conditions of symmetry will be satisfied:

$$
\begin{aligned}
& N_{x x}(x, y)=N_{x x}(-x, y), \quad N_{x y}(x, y)=-N_{x y}(-x, y), \\
& M_{x x}(x, y)=M_{x x}(-x, y), \quad M_{x y}(x, y)=-M_{x y}(-x, y), \quad V_{x}(x, y)=-V_{x}(-x, y),
\end{aligned}
$$

\footnotetext{
${ }^{3}$ It should perhaps be pointed out that (3.17) is a solution satisfying the differential equation (2.33), and is not the most general solution. However, with (3.17), since the solution thus found satisfies all the differential equations and, as will be shown, all the boundary conditions, it must be the solution of the physical problem.
} 
for the symmetric problem, and

$$
\begin{array}{ll}
N_{x x}(x, y)=-N_{x x}(-x, y), & N_{x y}(x, y)=N_{x y}(-x, y), \\
M_{x x}(x, y)=-M_{x x}(-x, y), & M_{x y}(x, y)=M_{x y}(-x, y), \quad V_{x}(x, y)=V_{x}(-x, y),
\end{array}
$$

for the antisymmetric problem. One may note that (4.1) and (4.2) are valid for all values of $x$ and $y$, and in the odd functions the discontinuity at $x=0$ may be allowed only on the crack surface; outside the crack all these functions (indeed, all field quantities) must be continuous. It is therefore clear that in solving the problem one needs to consider only one half of the cylinder, say $x>0$. Symmetry conditions similar to (4.1) and (4.2) are, of course valid for all the remaining field quantities. Thus, there are only five unknown functions $R_{1}, \cdots, R_{4}$, and $A_{1}$ which may be determined from five conditions specified at $x=$ +0 .

Consider now the symmetric problem for a circumferentially cracked shell. Noting that before the superposition which led to the perturbation problem the crack surfaces were free from all external loads, and since outside the crack all quantities are continuous, from (4.1) it may be concluded that

$$
N_{x y}(0, y)=0, \quad M_{x y}(0, y)=0, \quad V_{x}(0, y)=0, \quad-\infty<y<\infty,
$$

and $N_{x x}(+0, y)$ and $M_{x x}(+0, y)$ are known functions in $-1<y<1$. The problem is then a mixed boundary value problem. By using (4.3), three of the five unknowns $\left(R_{1}, \cdot, R_{4}, A_{1}\right)$ can be eliminated. The remaining two may be determined either from a system of dual integral equations or a system of singular integral equations arising from the mixed boundary conditions.

In terms of the normalized quantities the relevant force and moment resultants are given by

$$
N_{x x}=\partial^{2} \phi / \partial y^{2}, \quad N_{x y}=-\partial^{2} \phi / \partial x \partial y,
$$

$M_{x x}=\frac{a}{h \lambda^{4}}\left(\frac{\partial \beta_{x}}{\partial x}+\nu \frac{\partial \beta_{y}}{\partial y}\right), \quad M_{x y}=\frac{a}{h \lambda^{4}} \frac{1-\nu}{2}\left(\frac{\partial \beta_{x}}{\partial y}+\frac{\partial \beta_{y}}{\partial x}\right), \quad \frac{\partial V_{x}}{\partial y}=\frac{\partial^{2} w}{\partial x \partial y}+\frac{\partial \beta_{x}}{\partial y}$.

Using (4.4) and the results obtained in Secs. 2 and 3, for $x>0$ these quantities may be expressed in terms of $R_{1}, \cdots, R_{4}$ and $A_{1}$ as follows:

$$
\begin{aligned}
N_{x x}(x, y)= & -\frac{1}{2 \pi} \int_{-\infty}^{\infty}\left[\left(\frac{\lambda_{2}}{\lambda}\right)^{2} \alpha^{2} \sum_{1}^{4}\left(\frac{m_{j}}{p_{j}}\right)^{2} R_{j}(\alpha) \exp \left(m_{j} x\right)\right] \exp (-i y \alpha) d \alpha, \\
N_{x y}(x, y)= & \frac{i}{2 \pi} \int_{-\infty}^{\infty}\left[\left(\frac{\lambda_{2}}{\lambda}\right)^{2} \alpha \sum_{1}^{4} \frac{m_{j}^{3}}{p_{j}^{2}} R_{f}(\alpha) \exp \left(m_{j} x\right)\right] \exp (-i y \alpha) d \alpha, \\
M_{x x}(x, y)= & \frac{1}{2 \pi} \frac{a}{h \lambda^{4}}\left[\int_{-\infty}^{\infty} \sum_{1}^{4} \frac{m_{j}^{2}-\nu \alpha^{2}}{\kappa p_{j}-1} R_{f}(\alpha) \exp \left(m_{j} x\right) \exp (-i y \alpha) d \alpha\right. \\
& \left.-\frac{\kappa(1-\nu)^{2}}{2} \int_{-\infty}^{\infty} i \alpha r_{1} A_{1}(\alpha) \exp \left(r_{1} x\right) \exp (-i y \alpha) d \alpha\right], \\
M_{x y}(x, y)= & -\frac{1}{2 \pi} \frac{a(1-\nu)}{h \lambda^{4}} \int_{-\infty}^{\infty} i \alpha \sum_{1}^{4} \frac{m_{j} R_{f}(\alpha)}{\kappa p_{j}-1} \exp \left(m_{j} x\right) \exp (-i y \alpha) d \alpha \\
& -\frac{1}{2 \pi} \frac{a \kappa}{h \lambda^{4}} \frac{(1-\nu)^{2}}{4} \int_{-\infty}^{\infty}\left(\alpha^{2}+r_{1}^{2}\right) A_{1}(\alpha) \exp \left(r_{1} x\right) \exp (-i y \alpha) d \alpha,
\end{aligned}
$$




$$
\begin{aligned}
\frac{\partial}{\partial y} V_{x}(x, y)= & -\frac{1}{2 \pi} \int_{-\infty}^{\infty} i \alpha \sum_{1}^{4} \frac{\kappa p m_{j}}{\kappa p_{j}-1} R_{j}(\alpha) \exp \left(m_{j} x\right) \exp (-i y \alpha) d \alpha \\
& -\frac{1}{2 \pi} \kappa \frac{1-\nu}{2} \int_{-\infty}^{\infty} \alpha^{2} A_{1}(\alpha) \exp \left(r_{1} x\right) \exp (-i y \alpha) d \alpha
\end{aligned}
$$

We will attempt to solve the problem by reducing it to a system of singular integral equations. The problem is "symmetric" and the "normal" membrane and moment resultants are specified on the crack surface. Therefore, the natural "dual" quantities which should be considered as the new unknown functions are

$$
\frac{\partial}{\partial y} u(+0, y)=G_{1}(y), \quad \frac{\partial}{\partial y} \beta_{x}(+0, y)=G_{2}(y),
$$

corresponding to the "normal" displacement and rotation on the crack surface, respectively. In (4.10) the derivatives of the dual quantities are used to make them dimensionally consistent with $N_{x x}$ and $M_{x x}$ and in order to insure that the resulting integral equations will have Cauchy-type singularities [13]. The mixed boundary condition along $x=$ $0,-\infty<y<\infty$ may now be expressed as follows:

$$
\begin{gathered}
\lim _{x \rightarrow+0} N_{x x}(x, y)=F_{1}(y), \quad-1<y<1, \\
\lim _{x \rightarrow+0} M_{x x}(x, y)=F_{2}(y), \quad-1<y<1, \\
u(0, y)=0, \quad 1<|y|<\infty, \\
\beta_{x}(0, y)=0, \quad 1<|y|<\infty .
\end{gathered}
$$

Referring to the definitions (4.10) and the physical conditions (4.13) and (4.14), it is clear that the functions $G_{1}$ and $G_{2}$ must satisfy the following single-valuedness conditions:

$$
\int_{-1}^{1} G_{1}(y) d y=0, \quad \int_{-1}^{1} G_{2}(y) d y=0 .
$$

From (2.4), (2.14), (2.8), and (2.1) it may be shown that

$$
\frac{\partial^{2}}{\partial y^{2}} u(+0, y)=-\frac{\partial^{3}}{\partial x^{3}} \phi(+0, y)+\left(\lambda_{2} / \lambda\right)^{2} \frac{\partial}{\partial x} w(+0, y) \text {. }
$$

Then, after some manipulations, the quantities defined by (4.10) may be obtained as follows:

$$
\begin{gathered}
G_{1}(y)=\left(\frac{\lambda_{2}}{\lambda}\right)^{2} \frac{i}{2 \pi} \int_{-\infty}^{\infty} \frac{1}{\alpha} \sum_{1}^{4}\left(m_{j}-\frac{m_{j}^{5}}{p_{j}^{2}}\right) R_{j}(\alpha) \exp (-i y \alpha) d \alpha, \\
G_{2}(y)=-\frac{i}{2 \pi} \int_{\infty}^{\infty} \alpha \sum_{1}^{4} \frac{m_{j} R_{j}(\alpha)}{\kappa p_{j}-1} \exp (-i y \alpha) d \alpha-\frac{1}{2 \pi} \kappa \frac{1-\nu}{2} \int_{-\infty}^{\infty} \alpha^{2} A_{1}(\alpha) \exp (-i y \alpha) d \alpha .
\end{gathered}
$$


Now, using (4.3) and inverting (4.6), (4.8), (4.9), (4.17), and (4.18), we obtain five linear algebraic equations in $R_{1}, \cdots, R_{4}$, and $A_{1}$ which, after some modifications and by using (4.13) and (4.14), may be expressed as

$$
\begin{gathered}
A_{1}(\alpha)=2 \int_{-1}^{1} G_{2}(t) \exp (i \alpha t) d t, \\
\sum_{1}^{4} \frac{m_{j}^{3}}{p_{j}^{2}} R_{j}(\alpha)=0, \\
\sum_{1}^{4} \frac{m_{j}^{5}}{p_{j}^{3}} R_{j}(\alpha)=\frac{1-\nu}{\lambda_{2}{ }^{4}} i \alpha \int_{-1}^{1} G_{2}(t) \exp (i \alpha t) d t, \\
\sum_{1}^{4} \frac{m_{j}^{5}}{p_{j}^{4}} R_{j}(\alpha)=i \frac{\kappa}{\lambda_{2}{ }^{4}} \frac{1-\nu}{2 \alpha}\left(\alpha^{2}+r_{1}{ }^{2}\right) \int_{-1}^{1} G_{2}(t) \exp (i \alpha t) d t, \\
\sum_{1}^{4} \frac{m_{j}}{p_{j}^{2}} R_{j}(\alpha)=-i\left(\lambda / \lambda_{2}\right)^{2} \frac{1}{\alpha^{3}} \int_{-1}^{1} G_{1}(t) \exp (i \alpha t) d t .
\end{gathered}
$$

The solution of the system of equations (4.20) - (4.23) is given in Appendix B. By substituting from (4.19), Appendix B, (4.5) and (4.7) into (4.11) and (4.12), we obtain two integral equations to determine the unknown functions $G_{1}$ and $G_{2}$ of the following form:

$\lim _{x \rightarrow+0} \int_{-1}^{1} \sum_{1}^{2} G_{(}(t) d t \int_{-\infty}^{\infty} H_{i j}(\alpha, x) \exp (i(t-y) \alpha) d \alpha=F_{i}(y), \quad i=1,2,-1<y<1$.

By examining the functions $H_{i j}$, it can be seen that they are bounded everywhere in $-\infty<\alpha<\infty$. Therefore any possible singularity of the kernels in (4.24) at $y=t$ must be due to the behavior of $H_{i j}(\alpha, x)$ as $\alpha \rightarrow \mp \infty$. Note also that $H_{i j}$ contains exponential damping terms of the form $\exp \left(m_{j} x\right)$ and $\exp \left(r_{1} x\right)$, where $\operatorname{Re}\left(m_{j}\right)<0, \operatorname{Re}\left(r_{1}\right)<0$. However, since in the limit $x$ will go to zero, for $y=t$ this damping does not insure the convergence of the inner integrals in (4.24). The major difficulty in this problem, of course, is that the functions $m_{j}(\alpha)$ are not known explicitly in terms of $\alpha$. For the purpose of examining the singular behavior of the kernels in (4.24) and for extracting the singular parts, all one needs, however, is the asymptotic behavior of $m_{j}$ and $r_{1}$ as $|\alpha| \rightarrow \infty$. Thus, from (3.6) (3.8) and (3.15) it can be shown that for large values of $|\alpha|$ we have

$$
\begin{aligned}
& m_{j}(\alpha)=-|\alpha|\left(1+\frac{p_{j}}{2 \alpha^{2}}-\frac{p_{j}^{2}}{8 \alpha^{4}}+\cdots\right), \\
& r_{1}(\alpha)=-|\alpha|\left(1+\frac{1}{\kappa(1-\nu) \alpha^{2}}-\cdots\right) .
\end{aligned}
$$

Using now the relations (4.25) and (4.26) and separating the asymptotic values of $H_{i j}$ for large $|\alpha|$, the kernels in (4.24) may be expressed as

$$
\begin{aligned}
\int_{-\infty}^{\infty} H_{i j} \exp (i(t-y) \alpha) d \alpha=\int_{-\infty}^{\infty} H_{i j}^{\infty}(\alpha, x) \exp (i(t-y) \alpha) d \alpha \\
\quad+\int_{-\infty}^{\infty}\left[H_{i j}(\alpha, x)-H_{i j}^{\infty}(\alpha, x)\right] \exp (i(t-y) \alpha) d \alpha .
\end{aligned}
$$


where $H_{i j}{ }^{\infty}$ is the asymptotic value of $H_{i j}$ for $|\alpha| \rightarrow \infty$. On the right-hand side of (4.27) the first term gives Cauchy-type kernels $1 /(t-y)$ on the main diagonal terms, and the second integrals are uniformly convergent for all $t$ and $y$ (in which the limit $x=0$ can therefore be put under the integral sign). After the asymptotic analysis and some lengthy but straightforward manipulations the integral equations and the kernels may then be expressed as follows:

$$
\begin{aligned}
\int_{-1}^{1} \frac{G_{1}(t)}{t-y} d t+\sum_{1}^{2} \int_{-1}^{1} k_{1}(y, t) G_{j}(t) d t=2 \pi F_{1}(y), & -1<y<1 \\
& \frac{1-\nu^{2}}{\lambda^{4}} \int_{-1}^{1} \frac{G_{2}(t)}{t-y} d t+\sum_{1}^{2} \int_{-1}^{1} k_{2 j}(y, t) G_{j}(t) d t=2 \pi \frac{h}{a} F_{2}(y), \quad-1<y<1 \\
k_{11}(y, t) & =\int_{0}^{\infty}\left[\left(\frac{\lambda_{2}}{\lambda}\right)^{2} \alpha^{2} \sum_{1}^{4} \frac{2 m_{j}^{2}}{p_{j}^{2}} Q_{j}(\alpha)-1\right] \sin \alpha(t-y) d \alpha \\
k_{12}(y, t) & =2\left(\frac{\lambda_{2}}{\lambda}\right)^{2} \int_{0}^{\infty} \alpha^{2} \sum_{1}^{4} \frac{m_{j}^{2}}{p_{j}^{2}} N_{j}(\alpha) \sin \alpha(t-y) d \alpha \\
k_{21}(y, t) & =-\frac{2 \lambda_{2}^{4}}{\lambda^{4}} \int_{0}^{\infty} \sum_{1}^{4} \frac{1}{p_{j}^{4}}\left(m_{j}^{2}-\nu \alpha^{2}\right) m_{j}^{4} Q_{j}(\alpha) \sin \alpha(t-y) d \alpha \\
k_{22}(y, t) & =-2 \frac{1}{\lambda^{4}} \int_{0}^{\infty}\left[\sum_{1}^{4} \frac{\lambda_{2}{ }^{4}}{p_{j}^{4}}\left(m_{j}{ }^{2}-\nu \alpha^{2}\right) m_{j}^{4} N_{j}(\alpha)-\kappa(1-\nu)^{2} \alpha r_{1}+\frac{1-\nu^{2}}{2}\right] \sin \alpha(t-y) d \alpha
\end{aligned}
$$

where the functions $N_{f}(\alpha)$ and $Q_{j}(\alpha)$ are given in Appendix B. Using the results of Appendix B, it can be shown that $k_{12}(y, t)=k_{21}(y, t)$.

5. The asymptotic stress field around the crack tips. The solutions of the singular integral equations (4.28) and (4.29) have integrable singularities at the end points $y=\mp 1$ and are of the following form:

$$
G_{i}(y)=g_{i}(y)\left(1-y^{2}\right)^{-1 / 2}, \quad i=1,2,
$$

where $g_{1}$ and $g_{2}$ are bounded in $-1 \leq y \leq 1$. Similar to the plane problems, it can be shown that the behavior of the stress distribution in the immediate neighborhood of the crack tips is dependent on $g_{i}(\mp 1)$ only. To show this, one needs to substitute (5.1) through the expressions of $R_{1}, \cdots, R_{4}$ given in Appendix B and $A_{1}$ given by (4.19) into the original expressions for $N_{i j}$ and $M_{i j}(i, j=x, y)$, such as those given by (4.5)-(4.8). Using now the relation [14]

$$
\int_{0}^{\infty} \alpha^{\mu-1} \exp (-b \alpha)\left\{\begin{array}{l}
\sin \\
\cos
\end{array}\right\}(c \alpha) d \alpha=\frac{\Gamma(\mu)}{\left(b^{2}+c^{2}\right)^{\mu / 2}}\left\{\begin{array}{l}
\sin \\
\cos
\end{array}\right\}\left(\mu \tan ^{-1} \frac{c}{b}\right)(b>0, \quad \mu>0),
$$

and $[8,10,11]$ for large values of $|\alpha|$

$$
\begin{aligned}
\int_{-1}^{1} \frac{g(t)}{\left(1-t^{2}\right)^{1 / 2}} \exp (i \alpha t) d t=\left(\frac{\pi}{2|\alpha|}\right)^{1 / 2}\left\{g(1) \exp \left[i\left(\alpha-\frac{\pi}{4} \operatorname{sign}(\alpha)\right)\right]\right. \\
\left.+g(-1) \exp \left[-i\left(\alpha-\frac{\pi}{4} \operatorname{sign}(\alpha)\right)\right]+O\left(\frac{1}{|\alpha|}\right)\right\},
\end{aligned}
$$


around the end point $y=1, x=0$, the leading terms of the asymptotic stress and moment resultants may be expressed as

$$
\begin{aligned}
& N_{x x}(x, y) \cong \frac{g_{1}(1)}{2 \sqrt{2 \pi}} \int_{0}^{\infty} \frac{1}{\sqrt{ } \alpha}(1+\alpha|x|) \exp (-|x| \alpha) \sin \left[(1-y) \alpha-\frac{\pi}{4}\right] d \alpha, \\
& N_{y y}(x, y) \cong \frac{g_{1}(1)}{2 \sqrt{2 \pi}} \int_{0}^{\infty} \frac{1}{\sqrt{ } \alpha}(1-\alpha|x|) \exp (-|x| \alpha) \sin \left[(1-y) \alpha-\frac{\pi}{4}\right] d \alpha, \\
& N_{x y}(x, y) \cong \frac{g_{1}(1)}{2 \sqrt{2 \pi}} \int_{0}^{\infty} \sqrt{ } \alpha \exp (-|x| \alpha) \cos \left[(1-y) \alpha-\frac{\pi}{4}\right] d \alpha, \\
& M_{x x}(x, y) \cong \frac{g_{2}(1)}{2 \sqrt{2 \pi}} \frac{h}{12 a} \int_{0}^{\infty} \frac{1}{\sqrt{ } \alpha}(1+\alpha|x|) \exp (-|x| \alpha) \sin \left[(1-y) \alpha-\frac{\pi}{4}\right] d \alpha, \\
& M_{y y}(x, y) \cong \frac{g_{2}(1)}{2 \sqrt{2 \pi}} \frac{h}{12 a} \int_{0}^{\infty} \frac{1}{\sqrt{ } \alpha}(1-\alpha|x|) \exp (-|x| \alpha) \sin \left[(1-y) \alpha-\frac{\pi}{4}\right] d \alpha, \\
& M_{x y}(x, y) \cong \frac{g_{2}(1)}{2 \sqrt{2 \pi}} \frac{h}{12 a} \int_{0}^{\infty} \sqrt{ } \alpha x \exp (-|x| \alpha) \cos \left[(1-y) \alpha-\frac{\pi}{4}\right] d \alpha .
\end{aligned}
$$

From (5.4)-(5.9) it is seen that, aside from the magnitudes as represented by $g_{1}(1)$ and $g_{2}(1)$, the asymptotic behavior of the membrane and bending stress distributions around the crack tip will be identical. This is, of course, in agreement with the uncoupled in-plane and bending results for flat plates [4-7]. Defining the polar coordinates $r, \theta$ by

$$
x=r \sin \theta, y-1=r \cos \theta,
$$

evaluating the integrals, and observing that in dimenionsless quantities (see Appendix A)

$$
\frac{12 a z}{h} M_{i j}=\sigma_{i j}(i, j=x, y),
$$

from (5.4)-(5.9) the asymptotic stress distribution may be obtained as

$$
\begin{aligned}
& \sigma_{x x}(r, \theta, z) \cong-\frac{g_{1}(1)+z g_{2}(1)}{2(2 r)^{1 / 2}}\left[\frac{5}{4} \cos \frac{\theta}{2}-\frac{1}{4} \cos \frac{5 \theta}{2}\right], \\
& \sigma_{y y}(r, \theta, z) \cong-\frac{g_{1}(1)+z g_{2}(1)}{2(2 r)^{1 / 2}}\left[\frac{3}{4} \cos \frac{\theta}{2}+\frac{1}{4} \cos \frac{5 \theta}{2}\right], \\
& \sigma_{x y}(r, \theta, z) \cong-\frac{g_{1}(1)+z g_{2}(1)}{2(2 r)^{1 / 2}}\left[-\frac{1}{4} \sin \frac{\theta}{2}+\frac{1}{4} \sin \frac{5 \theta}{2}\right] .
\end{aligned}
$$

In this "symmetric" problem the stress intensity factor is defined by

$$
k_{1}=\lim _{X_{2} \rightarrow a}\left(2\left(X_{2}-a\right)\right)^{1 / 2} \sigma_{11}\left(0, X_{2}\right),
$$

which, referring to Appendix A and (5.12), may be obtained in terms of $g_{1}(1)$ and $g_{2}(1)$ as follows:

$$
k_{1}\left(X_{3}\right)=-\frac{E \sqrt{ } a}{2}\left[g_{1}(1)+\frac{X_{3}}{a} g_{2}(1)\right]
$$


It should be noted that even though the analysis given in the previous sections is valid for specially orthotropic as well as isotropic shells, (a) because of the dependence of $x$ and $y$ on the orthotropy constant $c=\left(E_{1} / E_{2}\right)^{1 / 4}, r$ and $\theta$ defined by (5.10) are not the physical coordinates, and consequently the angular distribution of the stress state in orthotropic shells would be different from that of isotropic shells as given by (5.12)-(5.14); and (b) since the roots $m_{j}$ and $r_{i}$ are very heavily dependent on $c$, the numerical results obtained for the isotropic shells by assuming $c=1$ cannot be readily adapted to orthotropic shells.

By performing the proper asymptotic analysis in the vicinity of the crack tip, similar results may be obtained for the transverse shear resultants. Consider, for example, the transverse shear resultant $V_{x}$. Noting that (4.9) is obtained from $V_{x}$ by direct differentiation, after some manipulations, for large values of $|\alpha|$ we obtain

$$
\begin{aligned}
V_{x}(x, y) \cong-\frac{1}{4 \pi} \kappa x \lambda_{2}{ }^{4} \int_{-\infty}^{\infty} \frac{1}{|\alpha|} & \exp (-|\alpha|-i \alpha y) d \alpha\left[\sum_{1}^{4} m_{j} R_{j}(\alpha)+i\left(\frac{\lambda}{\lambda_{2}}\right)^{2} \alpha \int_{-1}^{1} G_{1}(t) \exp (i \alpha t) d t\right] \\
& +\frac{i x}{2 \pi} \int_{-\infty}^{\infty} \frac{\alpha}{|\alpha|} \exp (-|\alpha x|-i \alpha y) d \alpha \int_{-1}^{1} G_{2}(t) \exp (i \alpha t) d t
\end{aligned}
$$

The leading term in the asymptotic expansion of $V_{x}$ in powers of $r$, the distance from the crack tip, is dependent on the behavior of its Fourier transform given in (5.17) for large values of $|\alpha|$. Now, from (3.5) and (3.10) we observe that $\sum m_{j} R_{j}$ is the Fourier transform of $\partial w / \partial x$ at $x=0$. Since $\partial w / \partial x$ at $x=0$ is bounded for all values of $y$, its Fourier transform must vanish as $|\alpha| \rightarrow \infty$. Therefore, the term $\sum m_{j} R_{j}$ in (5.17) would not have any contribution to the leading term in the asymptotic expansion of $V_{x}$.

Using now the expressions (5.1-5.3), from (5.17) near the end point $y=1, x=0$ we find

$$
V_{x}(x, y)=\frac{x}{(2 \pi)^{1 / 2}}\left[\frac{\kappa}{2} g_{1}(1)\left(\lambda \lambda_{2}\right)^{2}-g_{2}(1)^{*}\right] \int_{0}^{\infty} \alpha^{-1 / 2} \exp (-|x| \alpha) \sin \left[\alpha(1-y)-\frac{\pi}{4}\right] d \alpha .
$$

Or, substituting from (5.10) and evaluating the integral, (5.18) becomes

$$
V_{x}(r, \theta) \cong\left[-\frac{\kappa}{2} g_{1}(1)\left(\lambda \lambda_{2}\right)^{2}+g_{2}(1)\right]\left(\frac{r}{2}\right)^{1 / 2} \sin \theta \cos \frac{\theta}{2} .
$$

Note that in (5.19) $g_{1}$ and $g_{2}$ represent contributions of the in-plane and bending deformations to $V_{x}$. Thus, in a flat plate $\lambda_{2}=0$, the uncoupled membrane solution would have no contribution to $V_{x}$, and (5.19) would give the flat plate bending result which may be obtained from Reissner's theory [12].

6. Crack surface displacements. After obtaining the functions $G_{1}$ and $G_{2}$ upon solving the integral equations (4.28) and (4.29), through the expressions for $R_{1}, \cdots, R_{4}$, and $A_{1}$, any desired field quantity in the shell can be expressed in terms of $G_{1}$ and $G_{2}$ and can be easily evaluated. One such group of quantities of some practical interest is the components of crack surface displacement vector. In the symmetric problem under consideration, referring to the definition (4.10), the in-plane component of the crack surface displacement (i.e. the crack opening displacement) may easily be obtained as

$$
u(+0, y)=-\int_{y}^{1} G_{1}(t) d t,-1<y<1 .
$$


The component of the displacement vector which is perpendicular to the shell surface, i.e. $w(x, y)$, is given by (3.5) and (3.10). Expressing again $R_{1}, \cdots, R_{4}$ in terms of $G_{1}$ and $G_{2}$ (Appendix B), $w$ may be obtained as follows:

$$
\begin{aligned}
w(+0, y)= & -\frac{1}{\pi} \int_{-1}^{1} G_{1}(t) d t \int_{0}^{\infty} \sum_{1}^{4} Q_{j}(\alpha) \sin \alpha(t-y) d \alpha \\
& -\frac{1}{\pi} \int_{-1}^{1} G_{2}(t) d t \int_{0}^{\infty} \sum_{1}^{4} N_{j}(\alpha) \sin \alpha(t-y) d \alpha,
\end{aligned}
$$

where $Q_{j}$ and $N_{j}$ are given in Appendix B.

7. Numerical solution and results. The singular integral equations (4.28) and (4.29) subject to single-valuedness conditions (4.15) are solved by using Gauss-Chebyshev integration formulas [13]. As an example, a cracked cylindrical shell under uniform membrane and bending loads is considered. To make the practical applications of the results more convenient, these two loads are considered separately. The calculated results for the stress intensity factors are normalized with respect to the corresponding flat plate values. For example, if the applied loads are

$$
N_{11}\left(0, X_{2}\right)=-N_{11}=-h \sigma_{m}, M_{11}\left(0, X_{2}\right)=0,-a<X_{2}<a,
$$

the input functions in the integral equations (4.28) and (4.29) become

$$
F_{1}(y)=-N_{11} / h E, F_{2}(y)=0,
$$

and the corresponding flat plate stress intensity factor is $\sigma_{m} \sqrt{ } a$. Then the membrane and bending stress intensity ratios $k_{m m}$ and $k_{b m}$ are obtained from (5.16) as follows:

$$
\begin{aligned}
& k_{m m}=k_{1}(0) / \sigma_{m} \sqrt{ } a=-\frac{E}{2 \sigma_{m}} g_{1}(1), \\
& k_{b m}=\left(k_{1}(h / 2)-k_{1}(0) / \sigma_{m} \sqrt{ } a=-\frac{E}{2 \sigma_{m}} \frac{h}{2 a} g_{2}(1) .\right.
\end{aligned}
$$

The "bending stress intensity factor" is thus based on the bending stresses at the outer layer of the shell, $X_{3}=+h / 2$.

Similarly, if the external loads are

$$
N_{11}\left(0, X_{2}\right)=0, M_{11}\left(0, X_{2}\right)=-M_{11}=-\frac{h^{2}}{6} \sigma_{b},-a<X_{2}<a,
$$

then the input functions become

$$
F_{1}(y)=0, F_{2}(y)=-\left(M_{11} / E h^{2}\right),-1<y<1 .
$$

In this case too the corresponding flat plate stress intensity factor is defined by $\sigma_{b} \sqrt{ } a$, and the stress intensity factor ratios are obtained as follows:

$$
\begin{aligned}
& k_{m b}=k_{1}(0) / \sigma_{b} \sqrt{ } a=-\frac{E}{2 \sigma_{b}} g_{1}(1), \\
& k_{b b}=\left(k_{1}(h / 2)-k_{1}(0)\right) / \sigma_{b} \sqrt{ } a=-\frac{E}{2 \sigma_{b}} \frac{h}{2 a} g_{2}(1) .
\end{aligned}
$$


In the numerical calculations the effective transverse shear modulus for the shell is assumed to be $B=5 G / 6, G$ being the shear modulus of the material. Also, Poisson's ratio is taken as $\nu=1 / 3$ in all calculations except for one set of results where the effect of $\nu$ is investigated. One may note that $\lambda_{2}$ used in this analysis is the standard shell parameter $\lambda$ defined in the formulation of the problem by using the classical (i.e., the eight-order) shell theory. Also note that in the present analysis there is an additional parameter $a / h$, which, within the confines of the shallow shell theory, gives the thickness effect.

The numerical results are shown in Tables 1-5. Tables 1-4 show the stress intensity factor ratios $k_{m m}, k_{b m}, k_{m b}$, and $k_{s b}$ defined by (7.3), (7.4), (7.7), and (7.8), respectively. In these tables the values given for $\lambda_{2}=\left[12\left(1-v^{2}\right)\right]^{1 / 4} a /(R h)^{1 / 2}=0$ correspond to the flat plate. Both $\lambda_{2}$ and $a / h$ have been varied from 0 to 10 . For $\lambda_{2}>10$ and $a / h>10$ the linearized shallow shell theory used in this study is probably not valid. For very thin shells (i.e., $a / h=10$ ) under uniform membrane loading (Tables 1,2$)$ it is found that the membrane component of the stress intensity factor $k_{m m}$ is indistinguishable from the results found by using the classical theory [1]. However, the bending stress intensity factor $k_{b m}$ is quite different. The results given in Tables 1-4 show that, particularly for larger values of $\lambda_{2}$, the thickness parameter $a / h$ may have some effect on the stress intensity factors. Table 3 shows that when $\lambda_{2} \rightarrow 0$ the results are in good agreement with the flat plate bending results given in [5] and [6] and the axially cracked shell results given in [8]. Note that in Tables 14 the columns are truncated at roughly $\lambda_{2}=a / h$. Of course the reason for this is that for a fixed value of $a / h$, the values of $\lambda_{2}$ greater than those for which the stress intensity factors have been calculated would correspond to unrealistically large values of $h / R$.

For a specific geometry $\lambda_{2}=3$ and $a / h=5$, Table 5 shows the effect of the Poisson's ratio $\nu$ on the stress intensity factors. For the two most important components $k_{m m}$ and $k_{b b}$

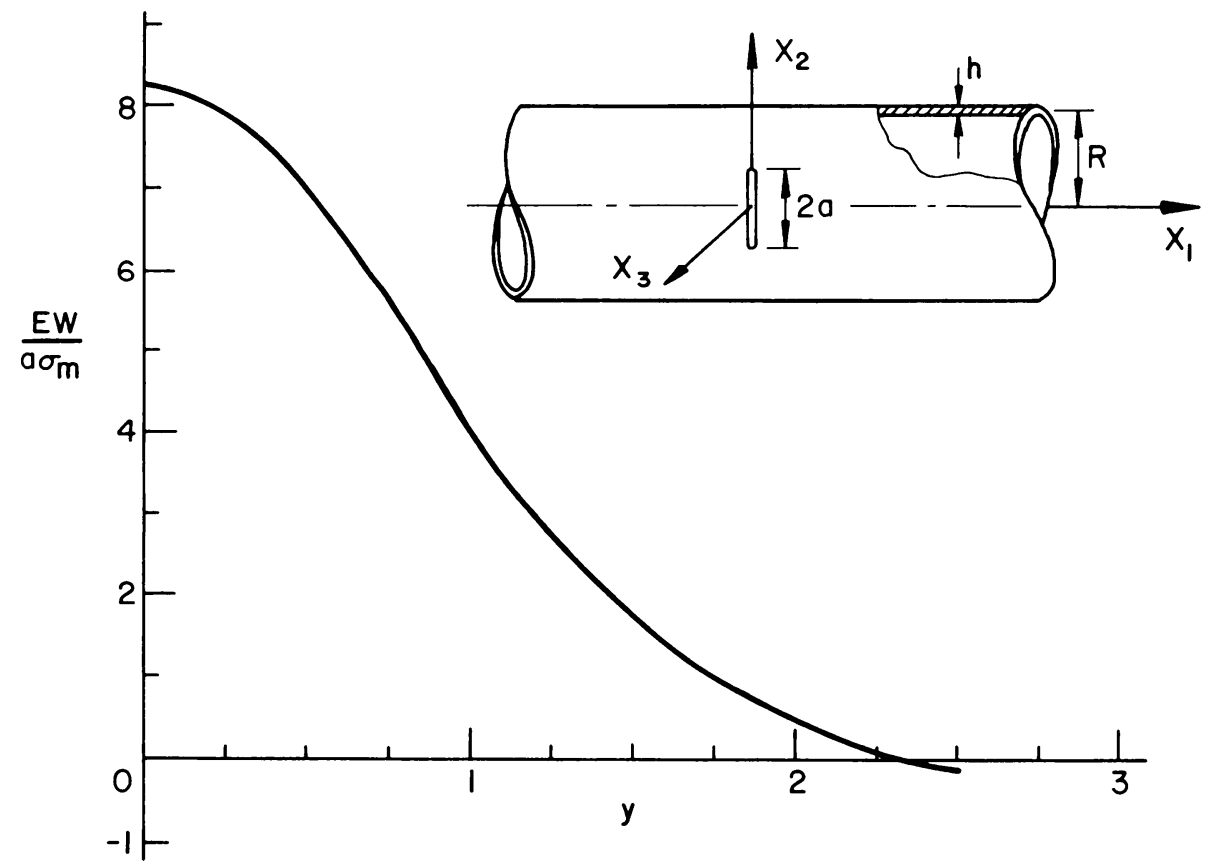

FIG. 1. Displacement component perpendicular to the shell surface in an isotropic cylinder, $N_{x x} \neq 0, M_{x x}=0$, $a / h=5, \lambda_{2}=3, \nu=1 / 3$. 


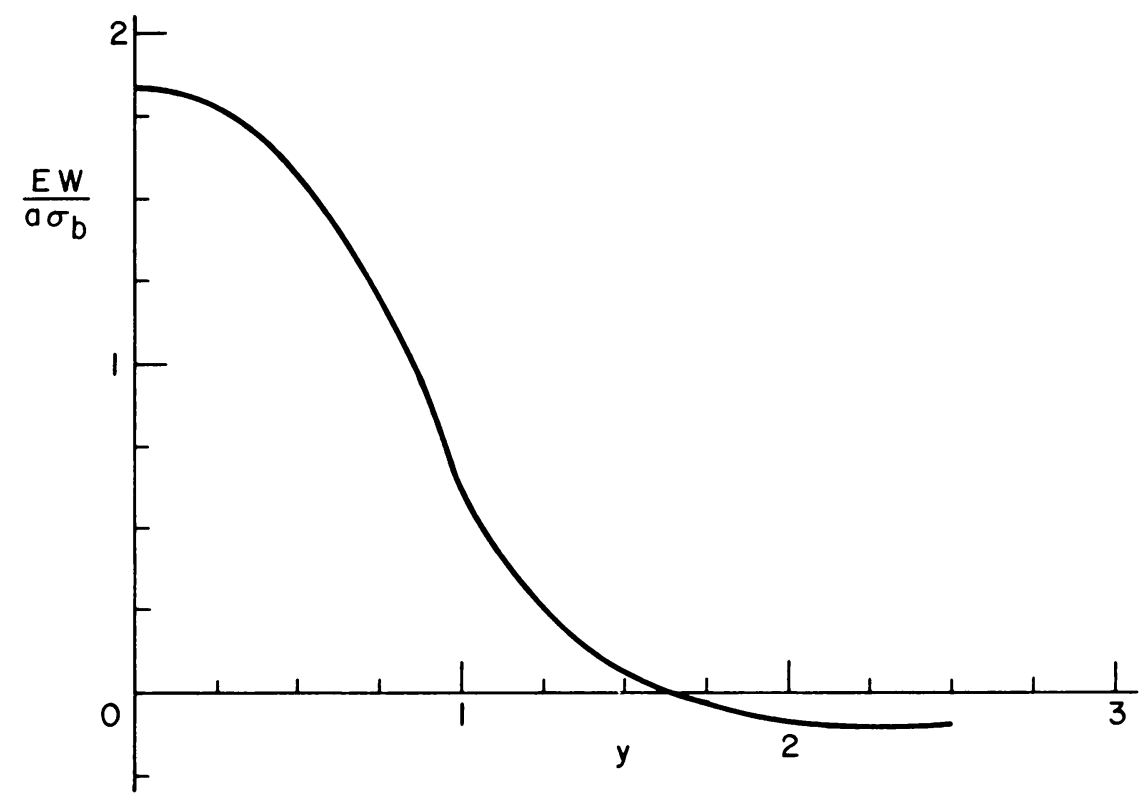

FIG. 2. Displacement component perpendicular to the shell surface in an isotropic cylinder, $N_{x x}=0, M_{x x} \neq 0$, $a / h=5, \lambda_{2}=3, \nu=1 / 3$.

(i.e., for the primary stress intensity factors under membrane and bending loads) the effect of $\nu$ does not seem to be significant. Hence the results given in Tables 1-4 and calculated for $\nu=1 / 3$ can be used for materials with a Poisson's ratio $0.2<\nu<0.4$, which may cover nearly all structural materials.

Figs. 1 and 2 show some sample results for the out-of-plane displacement $w(+0, y)$ calculated on the neutral surface along the line of the crack. The trend seems to be quite similar to the bulging results obtained for the axially cracked shell [11]. It should be emphasized that in these numerical calculations the crack surface membrane stress is compressive and the bending moment is applied in such a way that the outer layer of the shell on the crack surface is again compressive. Under these loads, as seen from the figures, on the crack surface the displacement $w$ is in the outward direction.

TABLE 1 .

Stress intensity factor ratio $k_{m m}(\nu=1 / 3)$

\begin{tabular}{rccccc}
\hline$\lambda_{2}$ & $\mathrm{a} / \mathrm{h}=0.5$ & $\mathrm{a} / \mathrm{h}=1$ & $\mathrm{a} / \mathrm{h}=2$ & $\mathrm{a} / \mathrm{h}=5$ & $\mathrm{a} / \mathrm{h}=10$ \\
\hline 0.0 & 1.000 & 1.000 & 1.000 & 1.000 & 1.000 \\
0.5 & 1.015 & 1.013 & 1.012 & 1.012 & 1.012 \\
1.0 & & 1.055 & 1.050 & 1.048 & 1.048 \\
1.5 & & & 1.108 & 1.103 & 1.102 \\
2.0 & & 1.179 & 1.169 & 1.168 \\
3.0 & & & 1.317 & 1.314 \\
4.0 & & & 1.467 & 1.462 \\
5.0 & & & 1.610 & 1.604 \\
6.0 & & & & 1.735 \\
8.0 & & & & \\
10.0 & & & & & 2.1870 \\
\hline
\end{tabular}


TABLE 2.

Stress intensity factor ratio $k_{b m}(\nu=1 / 3)$

\begin{tabular}{rccccc}
\hline$\lambda_{2}$ & $\mathrm{a} / \mathrm{h}=0.5$ & $\mathrm{a} / \mathrm{h}=1$ & $\mathrm{a} / \mathrm{h}=2$ & $\mathrm{a} / \mathrm{h}=5$ & $\mathrm{a} / \mathrm{h}=10$ \\
\hline 0.0 & 0.000 & 0.000 & 0.000 & 0.000 & 0.000 \\
0.5 & 0.048 & 0.044 & 0.043 & 0.041 & 0.041 \\
1.0 & & 0.093 & 0.092 & 0.092 & 0.092 \\
1.5 & & & 0.114 & 0.119 & 0.123 \\
2.0 & & 0.107 & 0.119 & 0.125 \\
3.0 & & & 0.057 & 0.071 \\
4.0 & & & -0.042 & -0.024 \\
5.0 & & & -0.143 & -0.126 \\
6.0 & & & & -0.220 \\
8.0 & & & & & -0.374 \\
10.0 & & & & & -0.493 \\
\hline
\end{tabular}

TABLE 3.

Stress intensity factor ratio $k_{b b}(\nu=1 / 3)$.

\begin{tabular}{rccccc}
\hline$\lambda_{2}$ & $\mathrm{a} / \mathrm{h}=0.5$ & $\mathrm{a} / \mathrm{h}=1.0$ & $\mathrm{a} / \mathrm{h}=2$ & $\mathrm{a} / \mathrm{h}=5$ & $\mathrm{a} / \mathrm{h}=10$ \\
\hline 0.0 & - & 0.752 & 0.704 & 0.667 & 0.652 \\
0.5 & 0.782 & 0.718 & 0.676 & 0.644 & 0.631 \\
1.0 & & 0.646 & 0.613 & 0.590 & 0.581 \\
1.5 & & & 0.542 & 0.526 & 0.520 \\
2.0 & & 0.481 & 0.466 & 0.463 \\
3.0 & & & 0.376 & 0.373 \\
4.0 & & & 0.322 & 0.317 \\
5.0 & & & 0.288 & 0.281 \\
6.0 & & & & 0.256 \\
8.0 & & & & & 0.225 \\
10.0 & & & & & 0.204 \\
\hline
\end{tabular}

TABLE 4.

Stress intensity factor ratio $k_{m b}(\nu=1 / 3)$

\begin{tabular}{rccccc}
\hline$\lambda_{2}$ & $\mathrm{a} / \mathrm{h}=0.5$ & $\mathrm{a} / \mathrm{h}=1$ & $\mathrm{a} / \mathrm{h}=2$ & $\mathrm{a} / \mathrm{h}=5$ & $\mathrm{a} / \mathrm{h}=10$ \\
\hline 0.0 & 0.000 & 0.000 & 0.000 & 0.000 & 0.000 \\
0.5 & 0.016 & 0.014 & 0.012 & 0.010 & 0.010 \\
1.0 & & 0.030 & 0.027 & 0.024 & 0.022 \\
1.5 & & & 0.037 & 0.034 & 0.032 \\
2.0 & & 0.042 & 0.039 & 0.037 \\
3.0 & & & 0.041 & 0.040 \\
4.0 & & & 0.39 & 0.037 \\
5.0 & & & 0.035 & 0.034 \\
6.0 & & & & 0.031 \\
8.0 & & & & 0.027 \\
10.0 & & & & 0.025 \\
\hline
\end{tabular}


TABLE 5.

The effect of Poisson's ratio on the stress intensity factors, $a / h=5, \lambda_{2}=3$.

\begin{tabular}{ccccc}
\hline$\nu$ & $k_{m m}$ & $k_{b m}$ & $k_{b b}$ & $k_{m b}$ \\
\hline 0.0 & 1.301 & 0.058 & 0.395 & 0.029 \\
0.1 & 1.305 & 0.061 & 0.395 & 0.033 \\
0.2 & 1.310 & 0.061 & 0.390 & 0.036 \\
0.3 & 1.315 & 0.058 & 0.381 & 0.040 \\
$1 / 3$ & 1.317 & 0.057 & 0.376 & 0.041 \\
0.4 & 1.321 & 0.051 & 0.366 & 0.043 \\
0.5 & 1.329 & 0.037 & 0.346 & 0.046 \\
\hline
\end{tabular}

TABLE 6.

Stress intensity factors for concentrated membrane and stress resultants on the crack surface, $a / h=5, \lambda_{2}=3$, $\nu=1 / 3$.

\begin{tabular}{lcc}
\hline & $\begin{array}{c}N_{11}=-\delta(y) \\
M_{11}=0\end{array}$ & $N_{11}=0$ \\
& 0.536 & \\
$k_{m m}$ & 0.007 & 0.018 \\
$k_{b m}$ & & 0.025 \\
$k_{b b}$ & & $M_{11}=-\frac{1}{6} \delta(y)$ \\
$k_{m b}$ & & \\
\hline
\end{tabular}

The results given in Tables $1-5$ and Figs. 1 and 2 are obtained for uniform crack surface pressure or bending moment. Needless to say, the problem can be solved for any arbitrary symmetric load distribution by appropriately specifying the input functions $F_{1}$ and $F_{2}$ in Eqs. (4.28) and (4.29). For example, Table 6 shows a sample result for concentrated membrane and bending stress resultants on the crack surface. The stress intensity factor ratio corresponding to $k_{m m}$ in a flat plate under a concentrated compressive load is $1 / \pi$ which is seen to be considerably lower than 0.536 calculated for the particular shell under consideration.

\section{REFERENCES}

[1] F. Erdogan and M. Ratwani, Fatigue and fracture of cylindrical shells containing a circumferential crack, Int. J. Fracture Mech. 6, 379-392 (1970)

[2] F. Erdogan and M. Ratwani, A circumferential crack in a cylindrical shell under torsion, Int. J. Fracture Mech. 8, 87-95 (1972)

[3] E. S. Folias, $A$ circumferential crack in a pressurized cylinder, Int. J. Fracture Mech. 3, 1-12 (1967)

[4] J. K. Knowles and N. M. Wang, On the bending of an elastic plate containing a crack, J. Math. and Phys. 39, $223(1960)$

[5] N. M. Wang, Effects of plate thickness on the bending of an elastic plate containing a crack, J. Math. and Phys. 47, 371 (1968)

[6] R. J. Hartranft and G. C. Sih, Effect of plate thickness on the bending stress distribution around through cracks, J. Math. and Phys. 47, 276 (1968)

[7] L. G. Copley and J. L. Sanders, Jr., A longitudinal crack in a cylindrical shell under internal pressure, Int. J. Fracture Mechanics 5, 117-131 (1969)

[8] S. Krenk, Influence of transverse shear on an axial crack in a cylindrical shell, Int. J. Fracture 14, 123-143 (1978)

[9] P. M. Naghdi, Note on the equations of shallow elastic shells, Quart. Appl. Math. 14, 331 (1956)

[10] U. Yuceoglu and F. Erdogan, A cylindrical shell with an axial crack under skew-symmetric loading, Int. J. Solids Structures 9, 347 (1973) 
[11] F. Erdogan, Crack Problems in cylindrical and spherical shells, in Plates and shells with cracks, G. C. Sih, ed., Noordhoff International Publishing, Leyden, pp. 161-199 (1977)

[12] E. Reissner, On bending of elastic plates, Quart. Appl. Math. 5, 55 (1947)

[13] F. Erdogan, Complex function technique, in Continuum physics, Vol. II, A. C. Eringen, ed., Academic Press, pp. 523-603 (1975)

[14] I. S. Gradshteyn and I. M. Ryzhik, Table of integrals, series and products, Academic Press (1965)

[15] M. Abramowitz and I. A. Stegun, Handbook of mathematical functions, Dover Publications (1965)

\section{Appendix A: The Dimensionless Quantities.}

$$
\begin{aligned}
& x=\frac{1}{\sqrt{ } c} \frac{X_{1}}{a}, \quad y=\sqrt{ } c \frac{X_{2}}{a}, \quad z=X_{3} / a, \\
& u=\sqrt{ } c \frac{U_{1}}{a}, \quad v=\frac{1}{\sqrt{ } c} \frac{U_{2}}{a}, \quad w=W / a, \\
& \beta_{x}=\sqrt{ } c \beta_{1}, \quad \beta_{y}=\frac{1}{\sqrt{ } c} \beta_{2}, \quad \phi=\frac{F}{a^{2} h E}, \\
& \sigma_{x x}=\sigma_{11} / c E, \quad \sigma_{y y}=c \sigma_{22} / E, \quad \sigma_{x y}=\sigma_{12} / E, \\
& N_{x x}=N_{11} / c h E, \quad N_{y y}=c N_{22} / h E, \quad N_{x y}=N_{12} / h E, \\
& M_{x x}=M_{11} / c h^{2} E, \quad M_{y y}=c M_{22} / h^{2} E, \quad M_{x y}=M_{12} / h^{2} E, \\
& V_{x}=V_{1} / \sqrt{ } \operatorname{ch} B, \quad V_{y}=\sqrt{ } c V_{2} / h B \\
& \lambda_{1}{ }^{4}=12\left(1-\nu^{2}\right) \frac{c^{2} a^{4}}{h^{2} R_{1}{ }^{2}}, \quad \lambda_{2}{ }^{4}=12\left(1-\nu^{2}\right) \frac{a^{4}}{c^{2} h^{2} R_{2}{ }^{2}}, \\
& \lambda_{12}{ }^{4}=12\left(1-\nu^{2}\right) \frac{a^{4}}{h^{2} R_{12}{ }^{2}}, \quad \lambda^{4}=12\left(1-\nu^{2}\right) \frac{a^{2}}{h^{2}}, \quad \kappa=\frac{E}{B \lambda^{4}} .
\end{aligned}
$$

Appendix B: Expressions for $R_{f}(\alpha), j=1, \cdots, 4$.

$$
\begin{aligned}
& R_{j}(\alpha)=i\left[Q_{j}(\alpha) \int_{-1}^{1} G_{1}(t) \exp (i \alpha t) d t+N_{j}(\alpha) \int_{-1}^{1} G_{2}(t) \exp (i \alpha t) d t\right], \\
N_{1}(\alpha)= & \frac{1}{D(\alpha)} \frac{m_{2} m_{3} m_{4}}{\left(p_{2} p_{3} p_{4}\right)^{2}}\left\{-\alpha \frac{1-\nu}{\lambda_{2}{ }^{4}}\left[m_{2}{ }^{2}\left(\frac{m_{3}{ }^{4}}{p_{3}{ }^{2}}-\frac{m_{4}{ }^{4}}{p_{4}{ }^{2}}\right)-m_{3}{ }^{2}\left(\frac{m_{2}{ }^{4}}{p_{2}{ }^{2}}-\frac{m_{4}{ }^{4}}{p_{4}{ }^{2}}\right)\right.\right. \\
& \left.+m_{4}{ }^{2}\left(\frac{m_{2}{ }^{4}}{p_{2}{ }^{2}}-\frac{m_{3}{ }^{4}}{p_{3}{ }^{2}}\right)\right]+\frac{\kappa}{\lambda_{2}^{4}} \frac{1-\nu}{2 \alpha}\left(\alpha^{2}+r_{1}{ }^{2}\right) \\
& \left.\cdot\left[m_{2}{ }^{2}\left(\frac{m_{3}{ }^{4}}{p_{3}}-\frac{m_{4}{ }^{4}}{p_{4}}\right)-m_{3}{ }^{2}\left(\frac{m_{2}{ }^{4}}{p_{2}}-\frac{m_{4}^{4}}{p_{4}}\right)+m_{4}{ }^{2}\left(\frac{m_{2}{ }^{4}}{p_{2}}-\frac{m_{3}{ }^{4}}{p_{3}}\right)\right]\right\},
\end{aligned}
$$




$$
\begin{aligned}
Q_{1}(\alpha)= & \frac{1}{D(\alpha)}\left(\frac{\lambda}{\lambda_{2}}\right)^{2} \frac{1}{\alpha^{3}} \frac{\left(m_{2} m_{3} m_{4}\right)^{3}}{\left(p_{2} p_{3} p_{4}\right)}\left[\left(\frac{m_{3}{ }^{2}}{p_{3}} \frac{m_{4}{ }^{2}}{p_{4}{ }^{2}}-\frac{m_{4}{ }^{2}}{p_{4}} \frac{m_{3}{ }^{2}}{p_{3}{ }^{2}}\right)\right. \\
& \left.-\left(\frac{m_{2}{ }^{2}}{p_{2}} \frac{m_{4}{ }^{2}}{p_{4}{ }^{2}}-\frac{m_{4}{ }^{2}}{p_{4}} \frac{m_{2}{ }^{2}}{p_{2}{ }^{2}}\right)+\left(\frac{m_{2}{ }^{2}}{p_{2}} \frac{m_{3}{ }^{2}}{p_{3}{ }^{2}}-\frac{m_{3}{ }^{2}}{p_{3}} \frac{m_{2}{ }^{2}}{p_{2}{ }^{2}}\right)\right] \\
D(\alpha)= & \frac{m_{1} m_{2} m_{3} m_{4}}{\left(p_{1} p_{2} p_{3} p_{4}\right)^{2}}\left\{\left(m_{1}{ }^{2}-m_{2}{ }^{2}\right)\left(m_{3}{ }^{2}-m_{4}{ }^{2}\right)\left(\frac{m_{1}{ }^{4} m_{2}{ }^{4}}{\left(p_{1} p_{2}\right)^{2}}+\frac{m_{3}{ }^{4} m_{4}{ }^{4}}{\left(p_{3} p_{4}\right)^{2}}\right)\right. \\
+ & \left(m_{1}{ }^{2}-m_{3}{ }^{2}\right)\left(m_{4}{ }^{2}-m_{2}{ }^{2}\right)\left(\frac{m_{1}{ }^{4} m_{3}{ }^{4}}{\left(p_{1} p_{3}\right)^{2}}+\frac{m_{2}{ }^{4} m_{4}{ }^{4}}{\left(p_{2} p_{4}\right)^{2}}\right) \\
+ & \left.\left(m_{1}{ }^{2}-m_{4}{ }^{2}\right)\left(m_{2}{ }^{2}-m_{3}{ }^{2}\right)\left(\frac{m_{1}{ }^{4} m_{4}{ }^{4}}{\left(p_{1} p_{4}\right)^{2}}+\frac{m_{2}{ }^{4} m_{3}{ }^{4}}{\left(p_{2} p_{3}\right)^{2}}\right)\right\}
\end{aligned}
$$

The expressions for $N_{2}$ and $Q_{2}$ are obtained from (B.2) and (B.3) by replacing the indices in $m_{i}$ and $p_{j}$ sequentially from $2,3,4$ to $3,4,1$. Similarly, for $N_{3}$ and $Q_{3}$ the indices are replaced by $4,1,2$, and for $N_{4}$ and $Q_{4}$ by $1,2,3$. 